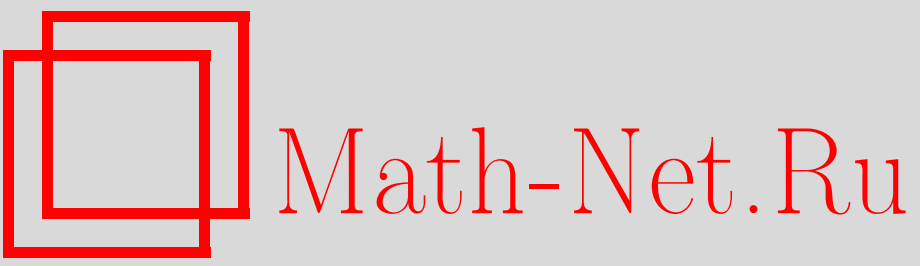

В. Н. Карпушкин, О числе компонент дополнения к некоторым алгебраическим кривым, УМН, 2002, том 57, выпуск 6, 185-186

DOI: https://doi.org/10.4213/rm584

Использование Общероссийского математического портала Math-Net.Ru подразумевает, что вы прочитали и согласны с пользовательским соглашением

http://www.mathnet.ru/rus/agreement

Параметры загрузки:

IP: 54.237 .206 .68

26 апреля 2023 г., 13:39:16 


\title{
О ЧИСЛЕ КОМПОНЕНТ ДОПОЛНЕНИЯ К НЕКОТОРЫМ АЛГЕБРАИЧЕСКИМ КРИВЫМ
}

\author{
В. Н. КАРПУШКИН
}

В работе [1] была дана верхняя оценка для числа компонент дополнения к нулям сферической функции (двух переменных) степени $n(n \geqslant 2)$. Здесь эта оценка несколько улучшается. Полученная оценка использует только некоторые простейшие геометрические свойства кривых - нулей сферических функций и поэтому является точной для более широкого класса кривых на проективной плоскости $\mathbb{R} P^{2}$ или на сфере $S^{2}$, называемых ниже допустимыми. Доказано, что число компонент дополнения к допустимой кривой на $\mathbb{R} P^{2}$ не превосходит $\left[(n-1)^{2} / 2\right]+2$. Указанная оценка сильнее, чем оценка Германа $n(n-1) / 2+1$ (см. [2]) для произвольных кривых, но слабее, чем оценка Гарнака $\left(n(n-3)+5+(-1)^{n}\right) / 2($ см. [3]) для неприводимых кривых. Данная работа уточняет результат работы [4].

1. Формулировки результатов. Пусть $S^{2}$ - стандартная сфера в $\mathbb{R}^{3}$ с индуцированной метрикой, $\mathbb{R} P^{2}$ - проективная плоскость с метрикой, построенной с помощью метрики на $S^{2}$. Кривой степени $n$ на $\mathbb{R} P^{2}$ или на $S^{2}$ назьвается множество нулей однородного многочлена степени $n$ от трех переменных.

Назовем вещественную нормализованную кривую $\Gamma$ на $\mathbb{R} P^{2}\left(S^{2}\right)$ допустимой, если из того, что прямые (большие окружности на $S^{2}$ ) $a$ и $b$ содержатся в $\Gamma$ и через их точку пересечения не проходят другие ветви кривой $\Gamma$, следует, что прямые $a$ и $b$ пересекаются ортогонально. Из вспомогательной леммы 1 работы [1] следует, что нули сферических функций - допустимые кривые.

Теорема. Пусть Г - допустимая кривая на $\mathbb{R} P^{2}$ степени $n, n \geqslant 2$. 1 . Предположим, что $n$ - четное число, тогда $\operatorname{dim} H_{0}\left(\mathbb{R} P^{2} \backslash \Gamma\right) \leqslant\left(n^{2}-2 n\right) / 2+2$. 2. Предположим, что $n$ - нечетное число, тогда $\operatorname{dim} H_{0}\left(S^{2} \backslash \Gamma\right) \leqslant(n-1)^{2} / 2+2$.

Ветвь кривой назьвается нечетной, если ее дополнение в $\mathbb{R} P^{2}$ состоит из односвязных кусков (см. [3]).

СледСтвиЕ. Пусть $\widetilde{\Gamma}-$ допустимая кривая на $S^{2}$ степени $n, n \geqslant 2$. 1. Предположим, что $n$ - четное число, тогда $\operatorname{dim} H_{0}\left(S^{2} \backslash \widetilde{\Gamma}\right) \leqslant n^{2}-2 n+4$. Если $n$ - четное число и кривая $\widetilde{\Gamma}$ не имеет нечетной ветви, то $\operatorname{dim} H_{0}\left(S^{2} \backslash \widetilde{\Gamma}\right) \leqslant n^{2}-2 n+3$. 2. Предположим, что $n$ - нечетное число, тогда $\operatorname{dim} H_{0}\left(S^{2} \backslash \widetilde{\Gamma}\right) \leqslant(n-1)^{2}+4$.

Далее везде предполагаем, что $\Gamma$ - допустимая кривая степени $n, \Gamma$ содержит $r$ различных вещественных прямых.

ЗАмечаниЕ. Оценки теоремы и следствия точны. Пусть $p$ - число неособых кривых 2-го порядка. Пусть все кривые, входящие в $\Gamma$, пересекаются только в вещественных точках. Тогда $\Gamma$ - связное подмножество $\mathbb{R} P^{2}$. Далее в этом замечании все особые точки кривой $\Gamma$ двойные. Через $\widetilde{\Gamma}$ обозначим кривую на $S^{2}$, соответствующую кривой $\Gamma$ на $\mathbb{R} P^{2}$. 1) Пусть $n=2 p$, $r=0$, кривая $\Gamma$ разбивает $\mathbb{R} P^{2}$ на один неодносвязньй кусок и односвязные куски, тогда $\left.\operatorname{dim} H_{0}\left(\mathbb{R} P^{2} \backslash \Gamma\right)=n(n-2) / 2+2, \operatorname{dim} H_{0}\left(S^{2} \backslash \widetilde{\Gamma}\right) \leqslant n(n-2)+3.2\right)$ Пусть $n=2 p+2$, $r=2$. Тогда кривая Г разбивает $\mathbb{R} P^{2}$ на односвязные куски и $\operatorname{dim}\left(\mathbb{R} P^{2} \backslash \Gamma\right)=n(n-2) / 2+2$, $\operatorname{dim}\left(S^{2} \backslash \widetilde{\Gamma}\right)=n(n-2)+4$. 3) Пусть $n=2 p+3, r=3$. Тогда кривая $\Gamma$ разбивает $\mathbb{R} P^{2}$ на односвязные куски и $\operatorname{dim} H_{0}\left(\mathbb{R} P^{2} \backslash \Gamma\right)=(n-1)^{2} / 2+2, \operatorname{dim} H_{0}\left(S^{2} \backslash \widetilde{\Gamma}\right)=(n-1)^{2}+4$. Заметим, что любые три прямые в $\mathbb{R} P^{2}$, лежащие в общем положении, проективным преобразованием переводятся в три ортогоналшные прямые.

Работа вьполнена при финансовой поддержке грантов NWO-РФФИ 047.008.005, INTAS-00259 . 
2. Доказательство теоремы. Далее предполагается, что $l$ - число $\mathbb{R}$-неприводимых кривых, составляющих кривую $\Gamma, k_{i}$ - число особых точек кривой $\Gamma$, в которых пересекаются по $i$ вещественных прямых $(i \geqslant 2)$ и, возможно, еще какие-либо ветви кривой $\Gamma$.

Лемма 1. Пусть Г - кривая на $\mathbb{R} P^{2}$ степени $n$. Тогда $\operatorname{dim} H_{0}\left(\mathbb{R} P^{2} \backslash \Gamma\right) \leqslant \frac{n^{2}-3 n}{2}+$ $q+l-\sum_{i \geqslant 3} k_{i} \frac{(i-1)(i-2)}{2}$. Здесь $q=2$, если $Г$ не имеет нечетной ветви, и $q=1$, если Г имеет нечетную ветвь.

Лемма 1 следует из теоремы Гарнака и теоремш Безу (см. [3]).

Лемма 2. Пусть Г - допустимая кривая степени п. Предположим, ито Г содержит $r$ пряммих, $r \geqslant 4$. Тогда $\sum_{i \geqslant 3} k_{i} \frac{(i-1)(i-2)}{2} \geqslant \frac{r(r-1)}{6}-\frac{r(r-1)^{1 / 2}}{6}$.

Докажем лемму 2.

ВСПОМОГАТЕЛЬНАЯ ЛЕМмА. Пусть Г - допустимая кривая, содержащая $r$ nрямых, $r \geqslant 4$. Пусть $m_{j}-$ число двойньх точек кривой $\Gamma$, которье суть двойные пересечения этих прямых с $j$-й прямой, $1 \leqslant j \leqslant r$. Тогда $m_{1}^{2}+\cdots+m_{r}^{2} \leqslant r(r-1)$ (у $j$-й прямой, принадлежащей кривой $\Gamma$, могут быть другие двойнье точки).

Доказательство вспомогательной леммы совершенно аналогично доказательству вспомогательной леммы 2 из работы [1]. Там используется условие ортогональности прямых, принадлежащих $\Gamma$.

Из формулировки вспомогательной леммы получим: $m_{1}+\cdots+m_{r} \leqslant r(r-1)^{1 / 2}$. Величина $\sum_{i \geqslant 3} k_{i} \frac{i(i-1)}{2}$ равна числу двойных точек, рожденных у кривой $\Gamma$ после приведения в общее положение всех прямых, входящих в кривую Г. Эти точки лежат на пересечениях пошевеленных прямых; $\frac{r(r-1)}{2}$ - число двойных точек $r$ прямых в общем положении. Здесь $\frac{r(r-1)^{1 / 2}}{2}-$ верхняя оценка на число двойных точек (до шевеления) у множества всех прямых (числом $r$ ), входящих в кривую Г. Отсюда получим $\sum_{i \geqslant 3} k_{i} \frac{i(i-1)}{2} \geqslant \frac{r(r-1)}{2}-\frac{r(r-1)^{1 / 2}}{2}$. Следовательно, $\sum_{i \geqslant 3} k_{i} \frac{(i-1)(i-2)}{2} \geqslant \frac{r(r-1)}{6}-\frac{r(r-1)^{1 / 2}}{6}$. Таким образом, лемма 2 доказана.

Заметим, что $l \leqslant \frac{n-r}{2}+r=\frac{n+r}{2}$. Теорема следует из лемм 1,2 при $r \geqslant 4$ и из леммы 1 (при $r \leqslant 3$ ).

Автор благодарен В.И. Арнольду, С. М. Гусейн-Заде и А. В. Чернавскому за полезные обсуждения.

\section{СПИСОК ЛИТЕРАТУРЫ}

[1] В. Н. Карпушкин // Функц. анализ и его прил. 1989. Т. 23. № 3. С. 59-60. [2] В. И. Арнольд // УМН. 1973. Т. 28. № 5. С. 260-262. [3] Д. А. Гудков // УМН. 1974. Т. 29. № 4. С. 3-79. [4] В.Н. Карпушкин // Функц. анализ и его прил. 1994. Т. 28. № 2. С. 52-54. 